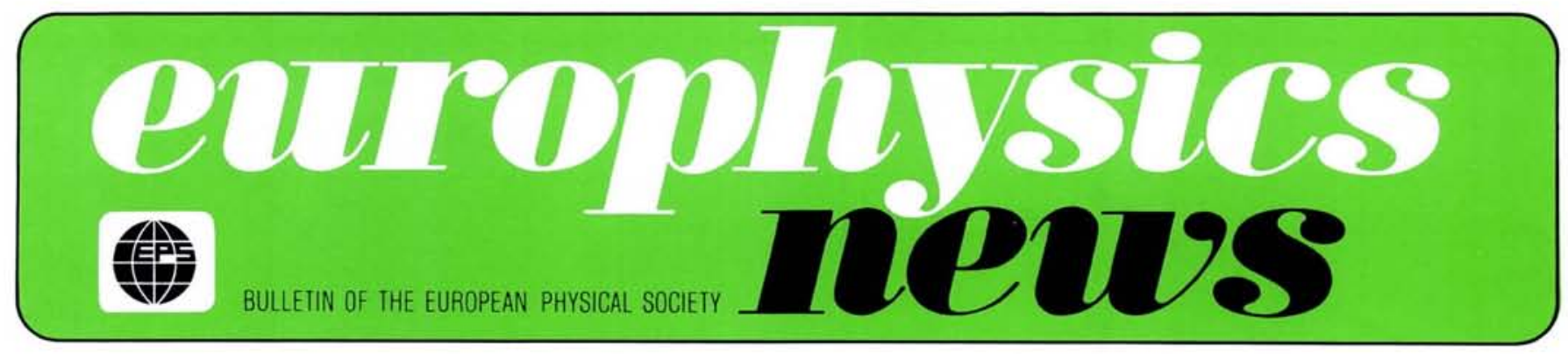

J.A.

Volume 14

Number 2

February 1983

\title{
Isotopic Anomalies Early History of the Solar System
}

\section{P. Bochsler, Bern}

(Physikalisches Institut, University of Bern)

The isotopic composition of various chemical elements in the Sun, planets and meteorites is one of the most important sources of information on the physical and chemical processes that shaped the solar system. For a long time, the presence of isotopes which show low natural abundances and are mainly produced by the decay of radioactive nuclides, has been used for dating events such as the solidification of minerals. Isotopes of noble gases have always been specially suited for such investigations since they are present in low concentrations in most natural samples. The most popular case is the pair ${ }^{40} \mathrm{~K} \rightarrow$ ${ }^{40} \mathrm{Ar}$; by far the largest part of ${ }^{40} \mathrm{Ar}$ present in the solar system is due to the decay of ${ }^{40} \mathrm{~K}$. More recently, with the continuing improvement of analytical techniques, it has been possible also to use systems in which the daughter isotope is originally present in nature in much larger fractions as with the ${ }^{87} \mathrm{Rb} \rightarrow{ }^{87} \mathrm{Sr}$ system.

Isotopes produced by decay of short lived, extinct isotopes have been used to compute time differences between the end of nucleosynthesis and solidification of parts of the solar system. The varying content of ${ }^{129} \mathrm{Xe}$ in meteorites generated from the decay of ${ }^{129} \mathrm{I}\left(T_{1 / 2}=15.7 \times 10^{6} \mathrm{a}\right)$, showed that the formation of the solar system, although it had not occurred in one instantaneous event, must have begun soon after the last nucleosynthetic event producing ${ }^{129} \mathrm{I}$. The combination of different dating techniques together with the development of efficient procedures for separating minerals from meteoritical samples down to microscopic scales, has led to rapid progress in our understanding of the events which took place during the early history of the solar system.

The underlying assumption for all dating methods was that the investigated material once had a uniform isotopic composition and that all deviations from this uniform composition had been caused by processes which happened after the separation of the solar system material from a larger, cosmic reservoir, billions of years ago. Processes considered were chemical fractionation, irradiation with energetic particles and radioactive decay.

Although for an amazingly large number of purposes this assumption can still be used as a working hypothesis, it has become now very clear that our original picture of an isotopically well-mixed early solar system no longer holds. There are two reasons which have caused the change of this view. First, the refinement of analytical procedures as a consequence of the Apollo missions to the Moon and the availability of lunar samples for investigation and, second, the fall of the ALLENDE-meteorite in 1969. ALLENDE, a carbonaceous meteorite of the rare type $\mathrm{C} 3 \mathrm{~V}$, contains large inclusions with minerals rich in refractory elements. The appearance of such minerals is expected for samples which have condensed at elevated temperatures above $1500 \mathrm{~K}$ and at low pressure from a gas of solar composition. These minerals are not common in normal meteorites and hence it looked promising to investigate "early condensates", thought of as the first solid witnesses of the birth of the solar system. The result of this investigation confirmed that the story of the early solar system was much more complicated than expected.

In the following, many elements in various classes of meteorites have shown evidence of incomplete mixing; isotopic anomalies have been found in the case of $\mathrm{O}, \mathrm{Ne}, \mathrm{Mg}, \mathrm{Si}, \mathrm{Ca}, \mathrm{Ti}, \mathrm{Kr}, \mathrm{Sr}, \mathrm{Te}, \mathrm{Xe}, \mathrm{Ba}$, $\mathrm{Nd}$ and are expected to be found also in other elements. It is not easy to give an adequate account of all the discoveries of the last few years, so we shall touch on a few outstanding cases only. It is appropriate to state at this point, that at present there is no canonic theory of the early chemical history of the solar system as it existed still a decade ago, as it is difficult to reconcile many of the pieces of evidence available today.

\section{Oxygen Anomalies in ALLENDE and Other Meteorites}

Oxygen has three stable isotopes. It is a rock forming element and its content in solid solar system materials is of the order of $40 \%$ by weight. The isotopic composition of oxygen is, therefore, extremely insensitive to any external influence such as irradiation of neighbouring target elements and there are no long lived radioactive nuclei decaying into oxygen isotopes. The only possible process affecting the isotopic composition of oxygen in a closed system is isotope fractionation by chemical or physical processes. These processes will always influence the ${ }^{18} \mathrm{O} /{ }^{16} \mathrm{O}$ ratio twice as strongly as the ${ }^{17} \mathrm{O} /{ }^{16} \mathrm{O}$ ratio and hence in a plot of ${ }^{17} \mathrm{O} /{ }^{16} \mathrm{O}$ vs. ${ }^{18} \mathrm{O} /{ }^{16} \mathrm{O}$ ratios, isotopic fractionation in a three-isotope system will be represented by points wandering along a straight line of slope $1 / 2$. This is depicted in Fig. 1 by the line labelled "terrestrial fractionated". In this figure and in the follow-

\section{Contents}

Isotopic Anomalies, Early History of the Solar System

Data Communications in High Energy Physics

Kenneth G. Wilson, Winner of the 1982 Nobel Prize in Physics

Spin Polarized Electrons

International Facilities for Physics Research 1

\section{4}

Hewlett-Packard Europhysics Prize 


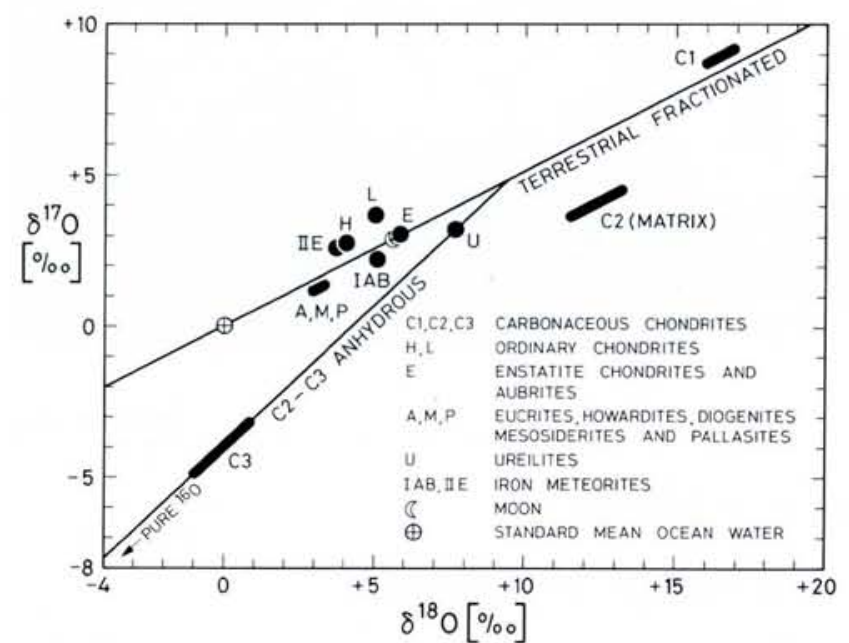

Fig. 1 - Oxygen isotope composition in solar system material. Adapted from R.N. Clayton (Phil. Trans. R. Soc. Lond. A, 303 (1981), 339-349). Isotope fractionation will appear as a point wandering along a line of slope 1/2 (e.g. "terrestrial fractionated"); mixture with a component enriched in pure 160 will appear as a line with slope 1 (e.g. $C_{2}-C_{3}$ anhydrous).

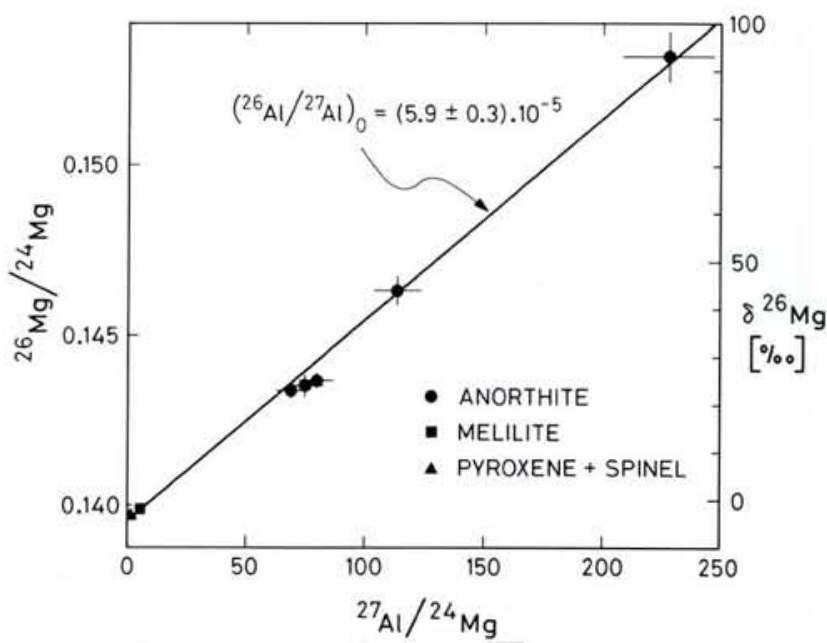

Fig. 2 - Magnesium isotope composition in different minerals from a Ca-Al-rich inclusion in the LEOVILLE carbonaceous meteorite (from Stegmann and Begemann, Earth Planet. Sci. Lett., 55 (1981), 266272). The straight line connects samples with an equal specific ${ }^{26} \mathrm{Al}$ activity during formation. ing we shall use the $\delta$-notation which is commonly used. It is defined by the expression:

$$
\delta^{18} \mathrm{O}=\frac{\left({ }^{18} \mathrm{O} /{ }^{16} \mathrm{O}\right)_{\text {sample }}-\left({ }^{18} \mathrm{O}{ }^{16} \mathrm{O}\right)_{\text {standard }}}{\left({ }^{18} \mathrm{O} /{ }^{16} \mathrm{O}\right)_{\text {standard }}} \times 1000 \%
$$

The common standard for oxygen isotopic composition is "Standard Mean Ocean Water" (SMOW).

As can be seen in Fig. 1, terrestrial and lunar samples as well as samples from two classes of meteorites, labelled by $E$, follow a fractionation line passing through SMOW. If solar system material had been separated from one reservoir and had only been affected by fractionation, all solar system samples should lie on this line or very close to it. Clayton, Grossman and Mayeda discovered in 1973 that some inclusions in the ALLENDE-meteorite and also samples from other carbonaceous meteorites did not fall on this fractionation line, but rather on a straight line with slope of nearly unity. In Fig. 1 this line is labelled "C2 - C3 anhydrous". The most extreme samples showed $-40 \%$ deviation in the ${ }^{18} \mathrm{O} /{ }^{16} \mathrm{O}$ ratio from the fractionation line. This observation could not be explained as a result of fractionation of what had been previously known as solar system oxygen and a new explanation was required.

Clayton, Grossman and Mayeda suggested this to be a mixture of a component enriched in pure ${ }^{16} \mathrm{O}$ and a component of fractionated "ordinary" oxygen. In a plot such as that shown in Fig. 1, any mixture of two components, each marked by a point in the plot, will lie on a straight line connecting the points. The exact location of the mixture point will depend on the relative contribution of the two components. Further investigations have shown that samples tend to pile up near the most extreme values found for ALLENDE, leading to the conclusion that the ${ }^{16} \mathrm{O}$-rich end member of the mixture had a composition not far from the investigated samples and must be very close to the most extreme ALLENDE points found so far. Despite the fact that, for instance, lunar and terrestrial samples are relatively widely spread along a fractionation line, many carbonaceous samples fall exactly on the $\mathrm{C} 2$ - C3 line, indicating that there was one single heavy type of oxygen present as a second component in many carbonaceous chondrites. Clayton and his coworkers, on the basis of additional observations, concluded that the ${ }^{16} \mathrm{O}$-rich component was probably present in the early solar nebula in solid form, possibly as interstellar dust, while the second component was gaseous.

It appears that this mixing event which formed oxygen in anhydrous phases of C2-and C3-carbonaceous meteorites must have taken place before the formation of other and different meteorites from the gaseous component. At present there is no simple, generally accepted theory on the nucleosynthetic origin of the observed oxygen anomalies; however, without complete understanding, evidence for generic relationships between different classes of meteorites and other planetary bodies is becoming available. For instance, it appears (Fig. 1) that the Moon, Earth and aubrites are closely related. Such a relationship clearly excludes certain models of the origin of the Moon.

\section{Magnesium and Extinct ${ }^{26} \mathrm{Al}$}

Magnesium has three stable isotopes, as does oxygen, its pecularity being that ${ }^{26} \mathrm{Mg}$ can be produced by the decay of ${ }^{26} \mathrm{Al}$ which has a half-life of $7.2 \times 10^{5} \mathrm{a}$.

After the discovery of oxygen anomalies in carbonaceous meteorites, Lee, Papanastassiou and Wasserburg found it worthwhile to investigate early condensates of ALLENDE for isotopic anomalies. They found a clear correlation of ${ }^{26} \mathrm{Mg}$ with the
$\mathrm{Al} / \mathrm{Mg}$ ratio. A typical example for such a correlation is shown in Fig. 2 which was obtained by Stegmann and Begemann from an analysis of different minerals in an inclusion of the LEOVILLE carbonaceous meteorite. Clearly this is what one would expect if ${ }^{26} \mathrm{Al}$ was included when the sample formed: ${ }^{26} \mathrm{Al}$ would be incorporated together with $\mathrm{Al}$ and after incorporation and solidification remain as ${ }^{26} \mathrm{Mg}$ predominantly in Al-rich phases. The straight line shown in Fig. 2 can then be interpreted as "isochrone", i.e. a line connecting samples of equal ${ }^{26} \mathrm{Al}$ concentrations or equal ages.

On the other hand, it should be noted that, as in the case of oxygen, the straight line in this three-isotope plot could also be interpreted as a mixing line, connecting two end members of different magnesium composition, one of them with normal magnesium, the other enriched in fossil ${ }^{26} \mathrm{Al}$. Hence a good correlation in such a plot as Fig. 2, does not directly prove that ${ }^{26} \mathrm{Al}$ was actively present when the sample solidified. Meanwhile, it has, however, been shown that a correlation also exists in cogenetic minerals, so that the two-component explanation can be excluded and there is almost no doubt left that ${ }^{26} \mathrm{Al}$ was indeed present in at least certain parts of the early solar system.

This observation implies two important facts:

- Solidification of some inclusions of LEOVILLE and also of ALLENDE occurred only a few million years after the material in those inclusions got a last admixture of freshly synthesized nuclei in non-negligible amounts.

- radioactive ${ }^{26} \mathrm{Al}$ has possibly been available as a powerful heat source during the early history of the solar system.

The question of the presence of a heat source is important for modelling the terrestrial planets (Venus, Earth, Moon, Mars) 
and the asteroids. In these bodies, $\mathrm{Al}$ is an abundant element and if an initial ${ }^{26} \mathrm{Al} /{ }^{27} \mathrm{Al}$ ratio was of the order of $10^{-5}$, this could cause melting of the inner parts of such a body. Owing to the relatively short half life of ${ }^{26} \mathrm{Al}$, the energy released from its decay in a sufficiently large body, of say $100 \mathrm{~km}$ radius, cannot be carried away by conduction, leading to elevated central temperatures and eventual melting of the inner parts. If the viscosity of the melting material is small enough and if the interior gravitational field is sufficiently strong, this will cause segregation of the body into a metallic core and a silicate mantle. Iron meteorites are considered to be fragments of such a metallic core of a small planet or an asteroid which broke up possibly because of a collision with another body during the history of the solar system.

The fact that ${ }^{107} \mathrm{Ag}$ anomalies, ascribed to the in situ decay of ${ }^{107} \mathrm{Pd}\left(T_{1 / 2}=6.5 \times\right.$ $10^{6}$ a) have been found in different iron meteorites, supports the importance of ${ }^{26} \mathrm{Al}$ as a potential powerful energy source for the early planets. Note, that while the case of ${ }^{26} \mathrm{Al}$ only links nucleosynthesis and the formation of $\mathrm{cm}$-sized inclusions, ${ }^{107} \mathrm{Pd}$ links nucleosynthesis and the existence of large segregated bodies to a short time scale of a few million years.

To complicate the picture, some, but not all ALLENDE inclusions do contain ${ }^{26} \mathrm{Mg}$ excesses. The presence of extinct ${ }^{26} \mathrm{Al}$ is not the only reason for inhomogeneities in the isotopic composition of magnesium. In some inclusions of ALLENDE, magnesium is also strongly mass-fractionated together with oxygen and silicon.

\section{Neon and Extinct ${ }^{22} \mathrm{Na}$}

It has been known for some time that neon in solar system material has more than one constituing component: "Spallogenic" neon produced by cosmic rays on elements abundant in meteorites $\mathrm{Mg}, \mathrm{Al}, \mathrm{Si}$ has the isotopic signature ${ }^{20} \mathrm{Ne}:{ }^{22} \mathrm{Ne} \cong$ $1: 1$. Solar wind neon is rich in ${ }^{20} \mathrm{Ne}$ with a ${ }^{20} \mathrm{Ne} /{ }^{22} \mathrm{Ne}$ ratio of 13.7 , while in terrestrial neon, this ratio is 9.8 only. In solar wind neon, the ${ }^{22} \mathrm{Ne} /{ }^{21} \mathrm{Ne}$ ratio is approximately
$31 \pm 4$ comparable with terrestrial atmospheric neon (34.5). Terrestrial neon, on the other hand, is comparable to "planetary" neon as observed in carbonaceous chondrites. In Fig. 3 these types are designed with "SP" for spallogenic, $\odot$ for solar wind, $\oplus$ for terrestrial and " $A$ " for planetary neon. As indicated by the dashed line in this figure, terrestrial and planetary neon could be explained as fractionated solar neon although the effect required is large and of the order of 40 percent. The reason for this is that neon as an element in meteorites and on the terrestrial planets is strongly depleted relative to the nonvolatile elements.

Again, in the three isotope plot in Fig. 3 , a binary mixture is represented as a straight line, a ternary mixture of solar wind neon, spallogenic neon and $\mathrm{Ne}-\mathrm{A}$ would lie within the solid triangle. A stepwise heating experiment by Jeffery and Anders on the ORGUEUIL carbonaceous meteorite showed that neon extracted between $800^{\circ} \mathrm{C}$ and $1100^{\circ} \mathrm{C}$ did not fall within this triangle and therefore Black concluded in 1972, that there must exist a component rich in ${ }^{22} \mathrm{Ne}$ which he called "Ne-E". Black hypothetically ascribed $\mathrm{Ne}-\mathrm{E}$ to be extra solar neon produced under nucleosynthetic conditions different from the normal solar system material. Subsequently, Eberhardt in 1974 and later Eberhardt, Niederer, Jungck and Meier succeeded in isolating $\mathrm{Ne}-\mathrm{E}$ rich carriers in micron sized grain separates from the ORGUEUIL meteorite and to limit the contents of ${ }^{20} \mathrm{Ne}$ and ${ }^{21} \mathrm{Ne}$ in $\mathrm{Ne}-\mathrm{E}$ to such low values that they could conclude that the separation of $\mathrm{Ne}-\mathrm{E}$ from other types of neon must be explained chemically. No natural physical process such as nucleosynthesis in supernovae or irradiation of a solid target with energetic particles would produce ${ }^{22} \mathrm{Ne}$ to such a high degree of purity. They suggest that $\mathrm{Ne}-\mathrm{E}$ is essentially fossil ${ }^{22} \mathrm{Na}$.

This has some astrophysically interesting consequences. If $\mathrm{Ne}-\mathrm{E}$ was incorporated into the solid grains in the form of ${ }^{22} \mathrm{Na}$, this must have happened very soon after production, since the half life of ${ }^{22} \mathrm{Na}$ is on-

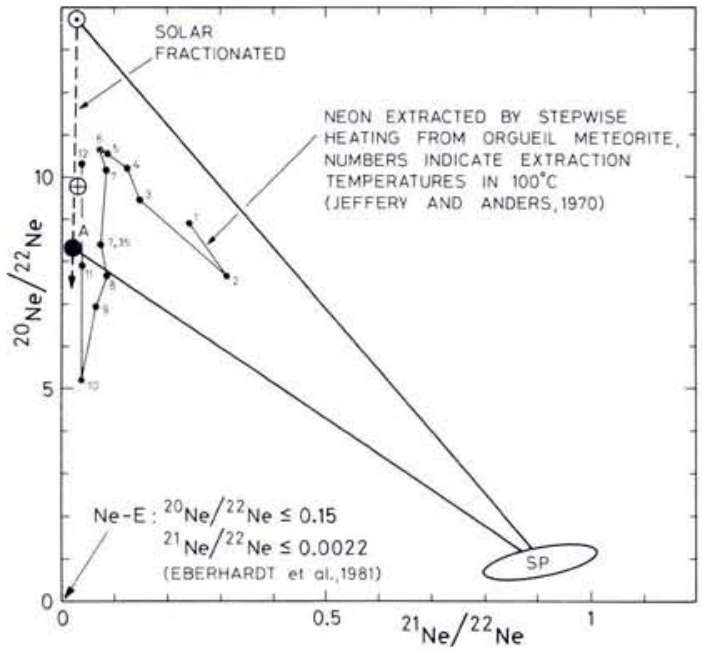

Fig. 3 - Isotopic composition of neon. The different types of neon are indicated:

$\odot=$ solar wind

$\oplus=$ terrestrial atmospheric,

$S P=$ spallogenic .

Ne-E from Eberhardt, Jungck, Meier and Niederer, Geochim, Cosmochim. Acta, 45 1981, 15151528. ly 2.6 a. Decay of ${ }^{22} \mathrm{Na}$ into ${ }^{22} \mathrm{Ne}$ must have taken place to a large extent after the grain carriers had released ${ }^{21} \mathrm{Ne}$ and ${ }^{20} \mathrm{Ne}$ which could have originally been incorporated as well. On the other hand, if ${ }^{22} \mathrm{Na}$ was produced by energetic irradiation of neighbouring elements $\mathrm{Mg}, \mathrm{Al}, \mathrm{Si}$ or $\mathrm{F}$ in the grains, this irradiation must have stopped before the closing of the carriers to $\mathrm{Ne}$-losses, otherwise $\mathrm{Ne}-\mathrm{E}$ would contain noticeable amounts of ${ }^{21} \mathrm{Ne}$ and ${ }^{20} \mathrm{Ne}$. This produces rather severe constraints on any astrophysical scenario which might explain the origin of the carriers. It should be noted, that they not only must survive the time between their formation and final incorporation into larger parts of the meteorite, but they also have to be kept at sufficiently low temperatures soon after separation from the nucleosynthetically active regions until now, in order to keep loosely bound ${ }^{22} \mathrm{Ne}$.

\section{Conclusion}

We have demonstrated three cases of isotopic anomalies, all of them showing that the solar system material has not been completely mixed prior to the formation of solid bodies. The incomplete mixing leaves us the possibility of looking more closely into the different processes of nucleosynthesis. This is more easily observed in the heavier elements with many isotopes and not directly linked to the origin of the solar system.

There is strong evidence for a very short time interval between nucleosynthesis and the onset of condensation. The close link of nucleosynthesis and star birth is independently supported by the astronomical observation that stars tend to form in clusters; massive stars and smaller stars like our Sun are born together. While massive stars lead a consumptive life and explode within a few million years after birth, smaller stars still in the process of formation in a cluster, might be contaminated with the nucleosynthetic debris of the more massive stars. Thus isotopic anomalies in solar system material can be viewed as the result of locally very fast recycling of interstellar matter. The thought of a close link between death and birth seems philosophically attractive, but we should keep in mind that such attractiveness can imply a dangerous intellectual bias.

\section{Further Reading}

Podosek F.A., 'Isotopic Structures in Solar System Materials', Ann. Rev. Astron. Astrophys. 16 (1978) 293-334.

Begemann F., 'Isotopic Anomalies in Meteorites', Rep. Prog. Phys. 43 (1980) 1309-1356.

Clayton R.N., 'Isotopic Variations in Primitive Meteorites', Phil. Trans. R. Soc. Lond. A 303 (1981) 339-349.

Wasserburg G.A., Papanastassiou D.A., and Lee T, 'Isotopic Heterogeneities in the Solar System', Early Solar System Processes and the Present Solar System, LXIII Corso, (Soc. Italiana di Fisica, Bologna) 1980 